MILa de MOURa Behar

PontRemol SALCEDO ${ }^{1}$

Gustavo Py Gomes DA SilverRA ${ }^{2}$

Cláudio Galeano Zettler ${ }^{3}$

Artigos originais

Palavras-chave

Neoplasia intra-epitelial cervical Neoplasias do colo do útero Condições pré-cancerosas Imunohistoquímica Genes p 16 Herpesvirus 2 humano

Keywords

Cervical intraepithelial neoplasia Uterine cervical neoplasm Precancerous conditions Immunohistochemistry Genes, pl6 Herpesvirus 2, human

\section{A expressão da proteína p16 e herpes simples vírus tipo 2 em lesões pré-neoplásicas e neoplásicas do colo do útero}

\author{
Immunobistochemical expression of $p 16$ and herpes simplex virus \\ type 2 in squamous intraepithelial lesions and cervical cancer
}

\section{Resumo}

OBJETIVO: demonstrar a expressão de biomarcadores, detectados por técnicas de imunohistoquímica, em tecidos sadios, lesões pré-neoplásicas e neoplásicas do colo do útero. MÉTODOS: para avaliação da reatividade imunohistoquímica de tecidos do colo do útero ao p 16 e ao herpes simples vírus tipo 2 (HSV-2), foram avaliadas 187 amostras de lesões intra-epiteliais de baixo grau (LIE-BG) e lesões intra-epiteliais de alto grau (LIE-AG) e carcinoma do colo do útero, e comparadas com grupo de pacientes sem lesões no colo uterino. A análise estatística foi realizada pelo teste do $\chi^{2}$ para tendências. $\bigcirc$ nível de significância foi de $\alpha=0,05$. RESULTADOS: foi avaliada a reatividade ao pl 6 com a seguinte distribuição: grupo sem lesão no colo do útero: 56\% (24/43), LIE-BG: 92\% (43/47), LIE-AG: 94\% (43/46) e câncer: 98\% (46/47) ( $p<0,001$, tendência linear). Com relação ao HSV-2: grupo sem lesão no colo do útero: 27\% (12/45), LIE-BG: 58\% (22/38), LIE-AG: 78\% (35/45) e câncer: 59\% (29/49) (p<0,001, tendência linear). Foi observado aumento na proporção de reatividade para os dois marcadores entre os grupos controle, LIE-BG, LIE-AG e câncer do colo do útero ( $p<0,001$ ). Não houve diferença significativa, quando comparamos apenas os grupos LIE-BG e LIE-AG entre si. CONCLUSÕES: foi verificado um aumento progressivo nas taxas de reatividade aos marcadores de imunohistoquímica estudados, com a severidade das lesões.

\section{Abstract}

PURPOSE: to demonstrate the expression of biomarkers, detected by immunohistochemical techniques in healthy tissues, as well as in preneoplastic and neoplastic lesions of the uterine cervix. METHODS: in order to evaluate the immunohistochemical reactivity of tissues from the uterine cervix to pl6 and to type 2 herpes simplex virus (HSV-2), 187 samples of low-grade intraepithelial lesions (LG-IEL) and high-grade intraepithelial lesions (HG-IEL), and of uterine cervix carcinoma were compared with a group of patients without uterine cervix lesions. Statistical analysis was done by the $\chi^{2}$ test for trends. The significance level was $\alpha=0.05$. RESULTS: the reactivity to p 16 was assessed showing the following distribution: group without uterine cervix lesions: 56\% (24/43), LG-IEL: 92\% (43/47), HG-IEL: 94\% (43/46), and cancer: 98\% (46/47) (p<0.001, linear trend). Concerning the HSV-2: group without uterine cervix lesions: 27\% (12/45), LG-IEL: 58\% (22/38), HG-IEL: 78\% (35/45), and cancer: $59 \%$ (29/49) $(p<0.001$, linear trend). There was an increase in the reactivity ratio for the two markers in the pathological groups (LG-IEL, HG-IEL and uterine cervix cancer, at $\mathrm{p}<0.001$ ) compared to controls. There was no significant difference between the LG-IEL and the HG-IEL groups. CONCLUSIONS: a progressive increase of reactivity ratios of the studied immunohistochemical markers as a function of lesion severity was observed.
Correspondência:

Mila de Moura Behar Pontremoli Salcedo Avenida América, 121/406 (EP 90440-020 - Porto Alegre/RS Fone: (51) 3342-3137/Fax: (51) 3338-7914 E-mail: mila_pontremoli@yahoo.com.br

Recebido $21 / 08 / 2006$

Aceito com modificacōes $12 / 02 / 2008$
Trabalho realizado na Fundação Universidade Federal de Ciências da Saúde de Porto Alegre, Faculdade de Medicina - UFCSPA Porto Alegre (RS), Brasil.

Professora do Departamento de Ginecologia e Obstetrícia da Fundação Universidade Federal de Ciências da Saúde de Porto Alegre - UFCSPA - Porto Alegre (RS), Brasil.

${ }_{2}^{2}$ Professor Titular de Ginecologia da Fundação Universidade Federal de Ciências da Saúde de Porto Alegre - UFCSPA - Porto Alegre (RS), Brasil.

3 Professor de Patologia da Fundação Universidade Federal de Ciências da Saúde de Porto Alegre - UFCSPA - Porto Alegre (RS), Brasil. 


\section{Introdução}

O câncer do colo do útero ainda é relevante, em termos de casos novos e de morte por câncer, principalmente em países em desenvolvimento ${ }^{1}$. Os programas de rastreamento para detecção de lesões precursoras e de câncer de cérvice baseiam-se na avaliação subjetiva da citologia com técnica de Papanicolaou ${ }^{2,3}$. A imunohistoquímica está cada vez mais sendo utilizada como auxiliar no diagnóstico em patologia cervical ${ }^{4}$. Esta técnica está conseguindo disponibilizar marcadores biológicos, o que deve contribuir para a melhora do rastreamento por definir critérios mais objetivos para identificar lesões pré-neoplásicas e neoplásicas do colo do útero ${ }^{2,5}$.

O vírus do papiloma humano (HPV) é considerado um importante fator na oncogênese cervical ${ }^{6-8}$. Estudos mostram que a integração do DNA viral ocasiona alteração na regulação da expressão dos oncogenes virais $\mathrm{E} 7$ e E6, que podem exercer seus efeitos, interferindo nas proteínas que regulam o crescimento celular, causando uma proliferação descontrolada, e, sendo assim, aumentando o risco de progressão das lesões intra-epiteliais (LIE) cervicais ${ }^{9-11}$.

O ciclo celular é regulado por uma seqüência de ativação e inativação de várias ciclinas-quinases dependentes em diferentes estágios do ciclo celular. Há vários pontos de controle ao longo do ciclo para checar a existência de algum dano celular que possa comprometer a síntese de DNA. Neste ponto a célula pode ser levada a apoptose (morte celular programada) ou mantida no ciclo celular. A passagem de G1 para S é determinada, entre outros eventos, pela ação da ciclina D e das CDKs 4 e 6 . Na fase $\mathrm{G} 1$, a ligação entre a ciclina $\mathrm{D}$ e CDK4 ou CDK6 irá fosforilar a proteína do retinoblastoma (pRB). Este evento provocará a liberação do E2F, fator de transcrição de genes essenciais à síntese de DNA, desencadeando a replicação do DNA e progressão para a fase $S$.

A proteína p16 está relacionada ao controle do ciclo celular, inativando sua progressão, por meio da inibição do complexo ciclina $\mathrm{D} / \mathrm{CDK} 4$ e 6 . A mutação ou deleção do gene p16 pode afetar o balanço entre a proteína p16 e ciclina $\mathrm{D}$, resultando em crescimento celular anormal (oncogênese) (32-14 $^{12}$ Carcinomas associados ao HPV são caracterizados por uma hiperexpressão do marcador p16 $6^{3,12}$, que é detectável pela imunohistoquímica. A proteína p16 tem sido proposta como um biomarcador que permite a identificação inequívoca das verdadeiras células displásicas e células neoplásicas, melhorando o diagnóstico específico ${ }^{12,14}$. Em um estudo atual, os autores referem que o p16 pode auxiliar a definir o diagnóstico dos casos inconclusivos entre lesão intra-epitelial de baixo grau (LIE-BG) e lesão intra-epitelial de alto grau (LIE-AG) ${ }^{15}$.
A demonstração imunohistoquímica de agentes infecciosos é útil na patologia ginecológica. Anticorpos contra proteínas virais como os utilizados para o herpes simples vírus (HSV) podem ser usados para demonstrar essas proteínas virais na citologia cervical e cortes de tecidos parafinados. Os vírus do herpes são vírus de DNA que replicam no núcleo da célula hospedeira, com a formação de inclusões intranucleares. A maioria dos casos de herpes genital é devida à infecção com o vírus herpes simples tipo $2(\mathrm{HSV}-2)^{16}$. Mais de $70 \%$ das infecções por HSV-2 são aparentemente assintomáticas ${ }^{16}$. É aceito atualmente que o HSV não apresenta papel central, mas é considerado como um co-fator em determinadas pacientes ${ }^{16}$. Acredita-se que vários tipos dos herpes vírus podem induzir transformações de células que estão em ciclo por meio da produção de proteínas ou pela replicação viral ${ }^{17}$.

Em nosso país, existem poucos estudos com esses marcadores tumorais em lesões pré-malignas e malignas do colo do útero. Essa investigação pretende demonstrar a expressão de biomarcadores, detectados por técnicas de imunohistoquímica, em tecidos sadios, lesões préneoplásicas e neoplásicas do colo do útero em uma amostra da população feminina do Brasil.

\section{Métodos}

Desenvolvemos um estudo transversal, contemporâneo. A amostra final foi constituída de 187 espécimes parafinados de pacientes, submetidas ao diagnóstico anátomo-patológico por biópsia de colo uterino, cirurgia de conização ou histerectomia, que foram arrolados de forma seqüencial, e analisados, no período de janeiro a dezembro de 2003. Os diagósticos foram de LIE-BG ou neoplasia intra-epitelial cervical (NIC) I (47 casos), LIE-AG ou NIC II-III (46 casos), carcinoma epidermóide do colo uterino (49 casos) e um grupo de pacientes sem lesão do colo do útero ( 45 casos). Foram excluídas do estudo pacientes que apresentaram mais de um diagnóstico anátomo-patológico divergente no colo uterino ou que apresentaram lesões diagnosticadas com anátomo-patológico na vagina ou na vulva concomitantes às lesões em estudo. Nos 187 casos examinados, alguns foram perdidos durante o processo de imunohistoquímica, por desgaste do material, ficando a amostra para expressão de p16 em 183 casos e para o herpes vírus em 177 casos.

Os controles de tecido de colo uterino sadio foram originados de pacientes submetidas a histerectomia sem lesões neoplásicas, todas com diagnóstico de liomioma uterino e sem lesões dignas de nota em colo uterino, e foram arrolados de forma consecutiva, a partir de janeiro de 2003. 
As pacientes foram também separadas em dois grupos, conforme a faixa etária: menos de 40 anos, 38,5\% (72 casos) do total da amostra; e idade igual ou maior de 40 anos, $61,5 \%$ (115 casos) do total da amostra.

A análise dos exames anátomo-patológicos e de imunohistoquímica foi realizada no Laboratório de Patologia do Programa de Pós-graduação em Patologia da Fundação Faculdade Federal de Ciências Médicas de Porto Alegre (FFFCMPA) e no Laboratório de Patologia do Complexo Hospitalar da Santa Casa de Porto Alegre (CHSCPA). A amostra foi classificada de acordo com as recomendações da Organização Mundial da Saúde (OMS) e do Colégio Americano de Patologistas (CAP) ${ }^{18}$.

\section{Método imunoistoquímico}

Os tecidos foram fixados em formalina e incluídos em parafina. Os blocos foram seccionados na espessura de $3 \mu \mathrm{m}$, desparafinados e reidratados para a realização da técnica. Foi utilizado o método estreptoavidina-biotinaperoxidase (kit LSAB + Peroxidase DakoCytomation ${ }^{\circledR}$ ) para detecção das proteínas p16 e HSV-2. A recuperação antigênica foi realizada com citrato de sódio $(\mathrm{pH} 6,0)$ aquecido em banho-maria $\left(95\right.$ a $\left.98^{\circ} \mathrm{C}\right)$ por 30 minutos. A atividade da peroxidase endógena foi bloqueada, utilizando-se dois banhos de dez minutos em peróxido de hidrogênio $\left(\mathrm{H}_{2} \mathrm{O}_{2}\right) 30 \mathrm{~V}$, a $5 \%$, em metanol. O bloqueio das proteínas inespecíficas foi realizado com soro normal de cavalo por 30 minutos. A incubação com os anticorpos primários realizou-se durante a noite, à temperatura de $4^{\circ} \mathrm{C}$. A incubação com anticorpo secundário e anticorpo terciário foi realizada durante 30 minutos à temperatura ambiente. Para controle negativo da técnica, foram utilizados os mesmos tecidos, incubados com os mesmos anticorpos - exceto o primário, que foi substituído por soro normal de cavalo. A ligação antígeno-anticorpo foi visualizada com o cromógeno diaminobenzidina (DAB). A contra-coloração foi feita com hematoxilina de Harris; as lâminas foram desidratadas e montadas com resina sintética. $\mathrm{O}$ anticorpo direcionado contra o antígeno p16 (p16 $6^{\mathrm{INK} 4 a}$, clone 6H12, Novocastra, Newcaster Upon, United Kingdom) foi utilizado na diluição $1 / 50$, e contra o antígeno HSV-2 (policlonal, DAKO Corporation, DK-2800 Glostrup, Denmark), na diluição 1/1500. Os controles positivos e negativos consistiram em tecido da vulva para o p16 e tecido cerebral para o HSV-2. As amostras foram avaliadas por dois observadores, que não tinham acesso às informações clínicas. Quando necessário, foi obtido o consenso entre os dois. Foram contados dez campos microscópicos de grande aumento (400X) e avaliou-se o percentual de núcleos e/ou citoplasmas positivos para o p16. Para a análise qualitativa, o p16 foi considerado positivo a partir de $1 \%$ de reatividade com intensa coloração nuclear. Com relação ao HSV-2, foi considerado positivo quando um núcleo fortemente corado por corte examinado foi detectado, tendo sido avaliada apenas a positividade ou não da reação.

\section{Análise estatística}

Os dados foram descritos, inicialmente, de forma quantitativa. Informações categorizadas quanto à positividade ou não dos marcadores foram expressas em percentual, sendo testadas por meio do teste do $\chi^{2}$ com aplicação adicional do teste de tendência linear. Para comparar distribuições de variáveis assimétricas entre dois grupos, foi utilizado o teste de Mann-Whitney. Para comparar variáveis categóricas com duas categorias, foi utilizado teste exato de Fisher. O nível de significância adotado foi $\alpha=0,05$. Os dados foram analisados com o programa Statistical Package for the Social Sciences (SPSS), versão 12.0. O projeto foi aprovado pelo Comitê de Ética em Pesquisa do CHSCPA e protocolado sob número 978/04 e parecer complementar número 329/04.

\section{Resultados}

A média de idade das 187 pacientes estudadas foi de 42,8 anos, com desvio padrão de 13,3. A idade mínima foi de 19 anos e a máxima foi de 86 anos.

Foi realizada uma comparação das idades das pacientes nos diferentes graus de lesão cervical, conforme apresentado na Tabela 1 . No grupo de LIE-BG havia $68 \%$ de pacientes com idades inferiores a 40 anos e no grupo LIE-AG foram 57\%, não sendo esses percentuais diferentes do ponto de vista estatístico. No grupo câncer, $18 \%$ das pacientes tinham idades inferiores a 40 anos, sendo este percentual diferente dos anteriores.

Os casos foram estudados em relação à presença dos dois biomarcadores p16 e HSV-2 nos diferentes grupos de graus de lesão. Foi avaliada a positividade para a proteína p16 e encontrou-se a seguinte distribuição: sem lesão: 56\% (24/43), LIE-BG: 92\% (43/47), LIE-AG: $94 \%$ (43/46) e câncer 98\% (46/47) (p<0,001). Com relação ao HSV-2, sem lesão: 27\% (12/45), LIE-BG: 58\% (22/38), LIE-AG: 78\% (35/45) e câncer: 59\% (29/49) (p<0,001). Os resultados constam da Tabela 2. Foi observada uma tendência de aumento na proporção de positividade do p16 entre os grupos sem lesão, LIE-BG, LIE-AG e câncer ( $\mathrm{p}<0,001$, tendência linear). $\mathrm{O}$ mesmo fenômeno foi observado para o HSV-2.

Quando os grupos foram estratificados por faixas etárias (maiores e menores de 40 anos), para o HSV-2 e o p16, a tendência de crescimento nas proporções de positividade dos grupos sem lesão ao câncer foi observada somente nas pacientes com mais de 40 anos, não sendo demonstrada nas pacientes com menos de 40 anos. Avaliou-se ainda a positividade para os dois biomarcadores separadamente 
nas duas faixas etárias. As pacientes mais jovens (menores de 40 anos) apresentaram maior reatividade ao p16 e ao HSV-2 do que as pacientes do outro grupo, com diferença significativa, conforme descrito na Tabela 3.

As proporções de reatividade para os dois marcadores estudados não apresentaram diferenças significativas, quando comparamos apenas os grupos LIE-BG e LIE-AG entre si.

No grupo LIE-BG, houve maior prevalência dos marcadores p16 e HSV-2 nas pacientes com menos de 40 anos de idade, porém essa diferença não foi significativa.

O grupo de casos de carcinoma epidermóide foi dividido por faixa etária, apresentando-se a grande maioria no grupo com idade igual ou maior que 40 anos $(81,6 \%)$. Entre essas pacientes com diagnóstico de câncer, verificamos que, para os dois grupos de idade (com ponto de corte em 40 anos), a freqüência da positividade dos dois marcadores de imunohistoquímica em questão foi semelhante.

Com relação ao marcador tumoral p16, de 183 casos, 156 foram positivos (85,2\%); 29,4\% (46) eram casos de câncer de colo uterino, $27,5 \%$ (43) eram casos de LIE-AG, 27,5\% (43) LIE-BG e 15,3\% (24) nos controles.

Encontrou-se a presença do HSV-2 em 98 casos $(55,3 \%$ do total da amostra). 29,6\% (29) nos casos de câncer,

Tabela 1- Comparação das idades entre os diferentes graus de lesão cervical

\begin{tabular}{lcc}
\hline Graus de lesão cervical & $<40$ anos $^{*}$ & $\geq 40$ anos \\
\hline LIE-BG $(n=47)$ & $32(68 \%)^{a}$ & $15(32 \%)$ \\
LIE-AG $(n=46)$ & $26(57 \%)^{a}$ & $20(43 \%)$ \\
Câncer $(n=49)$ & $9(18 \%)^{b}$ & $40(82 \%)$ \\
\hline
\end{tabular}

Dados apresentados como freqüências (percentuais). Na comparação, foi utilizado o teste do $\chi^{2}$ e comparações múltiplas; letras iguais representam percentuais iguais, letras diferentes representam percentuais diferentes ( $\left.{ }^{*} p<0,001\right)$. LIE-BG: lesão intra-epitelial de baixo grau; LIE-AG: lesão intra-epitelial de alto grau.

Tabela 2 - Comparação das frequiências dos marcadores nos diferentes graus de lesão

\begin{tabular}{lccccc}
\hline Marcadores & \multicolumn{4}{c}{ Graus de lesão } & \multirow{2}{*}{$\mathrm{p}$} \\
\cline { 2 - 5 } imunoistoquímicos & Sem lesĩo & LIE-BG & LIE-AG & Câncer & \\
\hline p16 & $\mathrm{n}=43$ & $\mathrm{n}=47$ & $\mathrm{n}=46$ & $\mathrm{n}=47$ & $<0,001^{*}$ \\
& $24(56 \%)$ & $43(92 \%)$ & $43(94 \%)$ & $46(98 \%)$ & \\
HSV-2 & $\mathrm{n}=45$ & $\mathrm{n}=38$ & $\mathrm{n}=45$ & $\mathrm{n}=49$ & $<0,001^{*}$ \\
& $12(27 \%)$ & $22(58 \%)$ & $35(78 \%)$ & $29(59 \%)$ & \\
\hline
\end{tabular}

Dados apresentados como freqüências (percentuais); na comparação foi utilizado o teste do $\chi^{2}$ para tendências. ${ }^{*}$ Foi considerada diferença significante $(p<0,05)$ para comparação entre o grupo sem lesões e os demais. LIE-BG: lesão intra-epitelial de baixo grau; LIE-AG: lesão intra-epitelial de alto grau; HSV-2: herpes simples vírus tipo 2.

Tabela 3 - Comparação das freqüências dos marcadores por faixa etária

\begin{tabular}{lccc}
\hline $\begin{array}{l}\text { Marcadores } \\
\text { imunoistoquímicos }\end{array}$ & $<40$ anos & $\geq 40$ anos & $p$ \\
\hline p16 & $\mathrm{n}=70$ & $\mathrm{n}=113$ & $0,031^{*}$ \\
& $65(93 \%)$ & $91(81 \%)$ & \\
HSV-2 & $\mathrm{n}=66$ & $\mathrm{n}=111$ & $0,002^{*}$ \\
& $47(71 \%)$ & $51(46 \%)$ & \\
\hline
\end{tabular}

Dados apresentados como freqüências (percentuais); na comparação foi utilizado o teste do $\chi^{2}$ para tendências. ${ }^{*}$ Foi considerada diferença significante $(p<0,05)$ para a comparação entre os grupos $<e \geq 40$ anos. HSV-2: herpes simples vírus tipo 2 .
$35,7 \%$ (35) LIE-AG, 22,4\% (22) LIE-BG e 12,2\% (12) nos controles. Em nenhum caso constatou-se a presença isolada de HSV-2 sem associação com o p16.

Constatou-se que, em 90 casos, houve associação de positividade do p16 e HSV-2, correspondendo a $52 \%$ dos casos (em 173 casos foi possível a análise desta associação).

Entre as pacientes com câncer invasor de cérvice uterina $27(57,4 \%)$ apresentaram positividade para $\mathrm{p} 16 \mathrm{e}$ HSV-2. Nas com lesão precursora, em 57 casos foi encontrada associação entre p16 e HSV-2 $(68,7 \%)$. No grupo sem lesão, nove pacientes apresentaram-se positivas para esses dois marcadores, correspondendo a 20,9\%.

\section{Discussão}

Nesse estudo, foi avaliada a positividade de marcadores de imunohistoquímica em lesões pré-invasoras e invasoras (carcinoma epidermóide) do colo do útero, comparando com tecido de cérvice uterina normal.

A proliferação celular é controlada por vários pontos que regulam o ciclo celular ${ }^{13}$. Acredita-se que a patogênese do carcinoma da cérvice uterina e suas lesões precursoras ocorram por meio de um processo com múltiplos fatores ${ }^{19}$. Sabe-se que o HPV é importante, considerado um dos principais agentes etiológicos, mas parece não ser suficiente para o desenvolvimento do câncer cervical ${ }^{9,20,21}$. Especial atenção tem sido dada aos cofatores, como outras infecções sexualmente transmissíveis e alterações genéticas ${ }^{9,19,22}$.

Nesse estudo, as pacientes foram divididas em faixas etárias com ponto de corte em 40 anos, já que, conforme dados dos Registros de Base Populacional do Instituto Nacional de Câncer (INCA), tanto o carcinoma in situ quanto o câncer invasor do colo uterino apresentam como maior incidência a faixa etária de 40 a 44 anos de idade ${ }^{1}$. Segundo alguns autores, a faixa etária preferencial do câncer de colo uterino é dos 40 aos $49 \operatorname{anos}^{23}$. A incidência máxima está sendo observada em uma faixa etária cada vez mais jovem: 40 a 45 anos. Isso representa a combinação do início mais precoce da atividade sexual (aquisição mais precoce da infecção por HPV) com os programas de triagem ativos que detectam câncer ou lesões pré-cancerosas em um momento mais cedo da vida ${ }^{24}$.

Avaliamos os dois grupos, conforme a faixa etária, e a maior parte das LIE ocorreu antes dos 40 anos de idade, com diferença significativa quando estas pacientes estão mais expostas aos diferentes fatores de risco, como maior atividade sexual, múltiplos parceiros e maior freqüência de infecções sexualmente transmissíveis, o que, possivelmente, explica porque as pacientes mais jovens apresentaram maior positividade ao p16, pela maior freqüência de infecção por HPV. Com relação ao câncer cervical invasor, verificou-se maior prevalência nas pacientes com idade maior ou igual a 40 anos, também com diferença estatística, concordando com a casuística encontrada ${ }^{1}$. 
Neste trabalho, foi observado um aumento progressivo na imunoexpressão isolada aos biomarcadores com a gravidade das lesões cervicais. Tais achados são semelhantes aos obtidos por outros autores ${ }^{7,14,15}$. Um estudo com 96 pacientes avaliou o p16, por imunohistoquímica, e a presença de HPV de alto risco, por captura híbrida, e concluiu que ambos os testes são complementares ao rastreamento das LIE-AG e auxiliam a definir o diagnóstico dos casos inconclusivos entre LIE-AG e LIE-BG ${ }^{15}$.

A hiperexpressão do p16 tem sido demonstrada em carcinomas relacionados ao HPV e lesões pré-malignas da cérvice uterina. Biomarcadores relacionados ao ciclo celular, como o p16, correlacionam-se com o grau de displasia das LIE cervicais ${ }^{7,11,14}$, como podemos evidenciar neste estudo com a tendência linear de aumento na expressão do p16 com o aumento da gravidade das lesões. Observou-se maior reatividade para o $\mathrm{p} 16$, nas pacientes mais jovens (menores de 40 anos), sendo essa diferença significativa.

Tem sido postulado que alguns agentes sexualmente transmissíveis, particularmente infecções virais, têm papel na carcinogênese da neoplasia cervical ${ }^{20}$. Embora as evidências sugiram a associação entre o HSV-2 e o desenvolvimento do câncer cervical invasivo, não há concordância entre os autores. Em um estudo com 25 casos de LIE-BG e 16 de LIE-AG, não foi encontrada positividade para HSV-2, porém não foram testados casos sem lesões cervicais ${ }^{8}$. Outros autores, por meio de uma metanálise, concluíram que infecção prévia ao HSV-2 não está associada a aumento de risco para desenvolvimento de câncer cervical, porém eles não descartam a possibilidade da neoplasia cervical predispor à infecção por HSV-2, tentando explicar a associação encontrada em outros estudos $^{25}$. Em outro trabalho, uma amostra com 200 casos de lesões invasoras e pré-invasoras do colo do útero foi avaliada por reação da polimerase em cadeia para HPV e HSV-2. Os autores não conseguiram demonstrar o papel do HSV-2 na carcinogênese cervical e sugerem que sejam realizados novos estudos para melhores esclarecimentos ${ }^{26}$. Neste estudo, em nenhuma paciente foi encontrado HSV-2 de forma isolada. Porém, quando associado com outro fator oncogênico, como o HPV, evidenciado pela hiperexpressão do p16, houve aumento na proporção de positividade com a gravidade das lesões do colo uterino. Nos casos de câncer, foi encontrado o HSV-2 em 59\% das pacientes, semelhante ao encontrado por um dos autores em estudo anterior (58\%) no mesmo Estado do Brasil (Rio Grande do Sul) ${ }^{7}{ }^{2}$

Em 52\% da amostra, encontramos associação positiva entre p16 e HSV-2, com maior prevalência entre as pacientes abaixo de 40 anos, com diferença significativa. As pacientes com lesão invasora ou pré-invasora apresentaram aproximadamente três vezes mais essa associação do que os casos com tecido cervical normal.

Foi verificado um aumento progressivo nas taxas de imunoexpressão dos marcadores estudados com a severidade das lesões. Por se tratar de estudo retrospectivo, com blocos de parafina antigos, não foi possível extrair o DNA do HPV. Nossos resultados sugerem que a expressão imunohistoquímica do p16 parece ser um instrumento importante na avaliação das lesões precursoras do câncer do colo do útero. Com relação ao HSV-2, continuam existindo dúvidas na literatura sobre o real papel do HSV-2 no câncer do colo do útero, entretanto a potencial relação entre essa virose e a patologia cervical aguarda outros estudos para melhores esclarecimentos.

\section{Referências}

1. Ministério da Saúde. Instituto Nacional de Câncer (INCA). Estimativa 2006: incidência de câncer no Brasil [ Internet]. Rio de Janeiro: INCA; 2005 [citado 2007 Abr 7]. Disponível em: http://www. inca.gov.br/estimativa/2006.

2. zur Hausen H. Papillomaviruses and cancer: from basic studies to clinical application. Nat Rev Cancer. 2002;2(5):342-50.

3. Ma L, Fisk JM, Zhang RR, Ulukus EC, Crum CP, Zheng W. Eosinophilic dysplasia of the cervix: a newly recognized variant of cervical squamous intraepithelial neoplasia. Am J Surg Pathol. 2004;28(11):1474-84

4. McCluggage WG. Immunohistochemistry as a diagnostic aid in cervical pathology. Pathology. 2007;39(1):97-1111.

5. Cox JT, Schiffman M, Solomon D; ASCUS-LSIL Triage Study (ALTS) Group. Prospective follow-up suggests similar risk of subsequent cervical intraepithelial neoplasia grade 2 or 3 among women with cervical intraepithelial neoplasia grade 1 or negative colposcopy and directed biopsy. Am J Obstet Gynecol. 2003;188(6):1406-12.
6. Cotran RS, Kumar V, Collins T. Neoplasia. In: Cotran RS, Kumar $V$, Collins T, editors. Robbins pathologic basis of disease. 6th ed. Philadelphia: WB Saunders; 1999. p. 260-327.

7. Vecchione A, Cermele C, Giovagnoli MR, Valli C, Alimandi M, Carico $E$, et al. p53 expression and genetic evidence for viral infection in intraepithelial neoplasia of the uterine cervix. Gynecol Oncol. 1994;55(3 Pt 1):343-8.

8. Golijow CD, Abba MC, Mourón SA, Laguens RM, Dulout FN, Smith JS. Chlamydia trachomatis and human papillomavirus infections in cervical disease in Argentine women. Gynecol Oncol. $2005 ; 96(1): 181-6$.

9. Kruse AJ, Skaland I, Janssen EA, Buhr-Wildhagen S, Klos J, Arends M, et al. Quantitative molecular parameters to identify low-risk and high-risk early $\mathrm{CIN}$ lesions: role of markers of proliferative activity and differentiation and Rb availability. Int J Gynecol Pathol. 2004;23(2):100-9.

10. Rivoire WA, Capp E, Corleta HE, Silva ISB. Bases biomoleculares da oncogênese cervical. Rev Bras Cancerol. 2001;47(2):179-84. 
11. O’Neill CJ, McCluggage WG. p 16 expression in the female genital tract and its value in diagnosis. Adv Anat Pathol. 2006;13(1):8-15.

12. Santos M, Montagut C, Mellado B, García A, Ramón y Cajal S, Cardesa A, et al. Immunohistochemical staining for p 16 and p53 in premalignant and malignant epithelial lesions of the vulva. Int J Gynecol Pathol. 2004;23(3):206-14.

13. Nogueira MC, Guedes Neto Ede P, Rosa MW, Zettler E, Zettler CG. Immunohistochemical expression of p16 and p53 in vulvar intraepithelial neoplasia and squamous cell carcinoma of the vulva. Pathol Oncol Res. 2006;12(3):153-7.

14. Focchi GR, Silva ID, Nogueira-de-Souza NC, Dobo C, Oshima CT, Stavale JN. Immunohistochemical expression of p16(INK4A) in normal uterine cervix, nonneoplastic epithelial lesions, and low-grade squamous intraepithelial lesions. J Low Genit Tract Dis. 2007; 11 (2):98-104.

15. Eleutério J Jr, Giraldo PC, Gonçalves AK, Cavalcante DI, de Almeida Ferreira FV, Mesquita SM, et al. Prognostic markers of high-grade squamous intraepithelial lesions: the role of pl6INK4a and high-risk human papillomavirus. Acta Obstet Gynecol Scand. 2007;86(1):94-8.

16. Azumi N, Czernobilsky B. Immunohistochemistry. In: Kurman R, editor. Blaustein's pathology of the female genital tract. 5th ed. Baltimore: Springer; 2002. p. 1251-76.

17. Lanham S, Herbert A, Basarab A, Watt P. Detection of cervical infections in colposcopy clinic patients. J Clin Microbiol. 2001;39(8):2946-50.

18. Branton PA. Protocol revision date: January 2005. Washington, D.C.: College of American Pathologists (CAP); 2005. [Based on WHO, AJCC/UICC, TNM 6th and FIGO Annual Report].

19. Hunt $\mathrm{CR}$, Hale RJ, Buckley $\mathrm{CH}$, Hunt J. p 53 expression in carcinoma of the cervix. J Clin Pathol. 1996;49(12):971-4.
20. Chan PK, Chan MY, Li WW, Chan DP, Cheung JL, Cheng AF. Association of human beta-herpesviruses with the development of cervical cancer: bystanders or cofactors. J Clin Pathol. $2001 ; 54(1): 48-53$

21. International Collaboration of Epidemiological Studies of Cervical Cancer. Carcinoma of the cervix and tobacco smoking: collaborative reanalysis of individual data on 13,541 women with carcinoma of the cervix and 23,017 women without carcinoma of the cervix from 23 epidemiological studies. Int J Cancer. 2006; $118(6): 1481-95$.

22. Bosch FX, Lorincz A, Muñoz N, Meijer CJ, Shah KV. The causal relation between human papillomavirus and cervical cancer. J Clin Pathol. 2002;55(4):244-65.

23. Pecorelli S, editor. FIGO Annual Report on the results of treatment in gynecological cancer. J Epidemiol Biostat. 2001;6:1-184.

24. Crum CP. The female and genital tract. In: Cotran RS, Kumar V, Collins T, editor. Robbins pathologic basis of disease. 6th ed. Philadelphia: WB Saunders; 1999. p. 329-402.

25. Lehtinen $M$, Koskela $P$, Jellum $E$, Bloigu $A$, Anttila $T$, Hallmans $G$, et al. Herpes simplex virus and risk of cervical cancer: a longitudinal, nested case-control study in the Nordic countries. Am J Epidemiol. 2002;156(8):687-92.

26. Pérez LO, Barbisan G, Abba MC, Laguens RM, Dulout FN, Golijow CD. Herpes simplex virus and human papillomavirus infection in cervical disease in Argentine women. Int J Gynecol Pathol. 2006;25(1):42-7.

27. Zettler CG. Detecção imuno-histoquímica do p53 no carcinoma epidermóide do colo do útero e sua associação com o papiloma viral humano e vírus herpes simples tipo 2 [tese]. Porto Alegre: Fundação Faculdade Federal de Ciências Médicas; 1996. 